\title{
Lessons from The Cultural and Political Economy of Recovery
}

\author{
Christopher J. Coyne Jayme S. Lemke $^{\dagger}$
}

\begin{abstract}
This essay reviews four of the central themes in Emily Chamily-Wright's The Cultural and Political Economy of Recovery. These themes include: (1) the cultural toolkit, (2) the use of qualitative methods in social science research, (3) polycentricism and disaster recovery, and (4) entrepreneurship in non-priced environments. Our purpose is twofold. First, to make clear Chamlee-Wright's contributions to our understanding of disaster recovery. Second, to demonstrate how these themes provide an opportunity for interdisciplinary exchange by blending insights from across the social sciences.
\end{abstract}

JEL Codes: B50, O10, O20, Z13

Keywords: cultural toolkit, disaster recovery, polycentricism, quantitative methods

\footnotetext{
¥ Email: CCoyne3@GMU.edu. Address: George Mason University, Department of Economics, MS 3G4, Fairfax, VA 22030

${ }^{\dagger}$ Email: Jlemke@GMU.edu. Address: George Mason University, Department of Economics, MS 3G4, Fairfax, VA 22030.
} 


\section{Introduction}

Hurricane Katrina's arrival at the Gulf Coast on August 29, 2005 was only the first in a chain of events that would change the face of the city for years to come. Between the dramatic beating from the storm and the second wave of damage from the broken levee, communities throughout the city suffered enormous damage. Destruction to property was estimated to total between $\$ 125$ and $\$ 165$ billion while 270,000 homes were ruined and 1,600 lives were lost (Chamlee-Wright 2010: 1). As Emily Chamlee-Wright's The Cultural and Political Economy of Recovery demonstrates, the responses of the communities within New Orleans in the days and months immediately following the disaster played an important role in determining how and indeed if these devastated communities would recover. ${ }^{1}$

At the theoretical level Chamlee-Wright's book seeks to better understand the nature of the relationship between market behavior and the cultural context within which exchange takes place. For this particular endeavor Chamlee-Wright borrows both methods and insights from anthropology, sociology, and economics to ask the question of how the cultural context of the various New Orleans communities affected market processes and social order. Her primary empirical tool is an extensive face-to-face interview process that enables Chamlee-Wright to gather, to the greatest extent possible, the in-depth local knowledge of those who actually experienced Katrina and its aftermath. The gathering of personal knowledge through the interview process allows Chamlee-Wright to illuminate the impact of culture on the broader recovery process.

\footnotetext{
${ }^{1}$ Chamlee-Wright's book is a part of the Mercatus Center's broader Crisis and Response in the Wake of Hurricane Katrina project which explored aspects of the post-Katrina recovery from a variety of perspectives (see Boettke et al. 2007).
} 
This essay reviews four of the central themes in The Cultural and Political Economy of Recovery: (1) the cultural toolkit, (2) the use of qualitative methods in social science research, (3) polycentricism and disaster recovery, and (4) entrepreneurship in non-priced environments. Our purpose is twofold. First, to make clear Chamlee-Wright's contributions to our understanding of disaster recovery. Second, to demonstrate how these themes provide an opportunity for interdisciplinary exchange by blending insights from across the social sciences. In what follows we dedicate a section to reach of the four themes. In each case we provide a brief overview of Chamlee-Wright's core arguments, as well as areas for future research and exploration.

\section{The Cultural Toolkit}

At the outset, Chamlee-Wright describes her project as an exercise in cultural economy, a research program that "seeks to understand why and how market processes work within specific cultural contexts" (Chamlee-Wright 2010: 16). The key intellectual progenitors of this research program are a diverse and multi-disciplinary group including: classical economist Adam Smith, political theorist Alexis de Tocqueville, new institutional economist Douglass North, and cultural sociologist Ann Swidler. The intellectual core shared by all of these scholars, including Chamlee-Wright, is the notion that the context within which a society operates will influence the functioning of the market and other social processes.

For Chamlee-Wright, socio-economic outcomes are the result of complex interactions between culture and the institutional rules of the community in question. She proposes that these interacting processes can be divided into four categories, each of which affects and is affected by 
every other: generalized norms, social networks, shared mental models, and cultural tools. In combination these processes form "the structure of socially embedded resources" (ChamleeWright 2010: 16).

Generalized norms and social networks are highly similar phenomena, with both seen as generally arising from repeated mutually beneficial social interaction. Their compatibility is illustrated with the example that a social network of individuals who grow to trust each other can lead to a general social norm of trust in exchange. Mental models and cultural tools are also similar in nature. In the attempt to distinguish these phenomena Chamlee-Wright offers up the complementary paradigms of culture as context versus culture as toolkit. Culture as context is the notion that shared mental models shape how we interpret the events and behaviors occurring around us. Culture as toolkit, in contrast, goes further by positing that individuals actively use this shared understanding of the world in order to create and implement better solutions to problems. For example, culture as context might form an individual's belief that spitting in an interview is bad, but actively using that knowledge to behave well during an interview and get a better job would be using culture as a tool.

The consideration of institutions_-cultural and legal, explicit and underlying, formal and informal-has moved to the forefront in the analysis of economic and social processes. Scholars in sociology and anthropology have long recognized the importance of these issues for understanding society. In economics, however, these issues are relatively new. That said, they have not been altogether neglected as illustrated by the work of F.A. Hayek, Deirdre McCloskey, and Douglass North, among others. Further, issues of culture and belief formation are no longer outside of mainstream consideration as illustrated by the work of Di Tella, Galiana, Schargrodsky (2007). Given the widespread recognition of culture and belief formation, the 
virtue of creating a new and distinct cultural toolkit cannot only be the recognition of culture in economic analysis. It must be something more.

The primary difference between the cultural toolkit and other institutional frameworks from our perspective is its process-oriented nature. Traditional institutional analysis, particularly where it is empirically driven, trends more towards the paradigm of comparative statics. This approach views institutions, and the play within those institutions, as equilibrium outcomes (see Ostrom 1986). However, as Boettke (1989) points out, human action is a process of continuous change and evolution that is best captured by an evolutionary rather than by an equilibrium economics. By allowing for interactivity between individual action and the resultant cultural context, the cultural toolkit utilized by Chamlee-Wright (2010) enables culture to be considered as a continuously evolving process that both shapes and is shaped by human action. This distinction imbues the cultural toolkit with both a distinct focus and a unique contribution to the field of institutional analysis.

If there is a complaint to be had with the cultural toolkit it is in the rhetorical complexity of the vocabulary offered, particularly given that it does not seem to play much of a role in the subsequent analysis. For example, one figure (2010: 19) proposes twelve different types of interactions between the four different categories of phenomena contained within the structure of socially embedded resources. However the subsequent analysis does not seem to depend much upon these rigid classifications. In other cases, Chamlee-Wright's use of concepts such as "social embeddedness" and "social capital" are too broadly defined running the risk of losing analytical tractability. To the extent that there is analytical value in distinguishing between these categories, demonstrating that value through explicit application could be an avenue for future research. 


\section{Qualitative Methods}

In addition to employing an analytical framework developed in the intersection of economics and sociology, Chamlee-Wright selects research methods located in the overlap of these disciplines. Though data from interview sources may be more commonly associated with anthropology and sociology than traditional economic research, Chamlee-Wright makes a compelling case for capturing in depth local knowledge and individual experiences as a way to better understand processes of discovery and exchange. Indeed, one of the key contributions of the book is that it provides a challenge to the traditional methods employed by economists to understand their subject of study.

At the empirical core of The Cultural and Political Economy of Recovery is a series of interviews conducted on the ground in New Orleans. Three hundred subjects were interviewed by Chamlee-Wright's team over a period of time beginning in February 2006 and concluding near the three year anniversary of Katrina. These first-hand accounts span parishes and cities, enabling identification of cultural attributes unique to particular communities, disaster response strategies, and recovery outcomes. The dominant cultural themes are identified by coding interview transcripts and categorizing the responses. Through this process patterns emerge, and local knowledge that was once completely dispersed has now been shaped in a manner enabling analysis.

The introduction of qualitative methods by Chamlee-Wright is a significant contribution in itself precisely because such techniques are rarely used in economic analysis. Through the interview process, Chamlee-Wright is able to attach specific meaning which could very well be 
lost through aggregate data. Moreover, qualitative methods allow her to trace the causal processes that cannot be teased out of correlations. Consider the New Orleans residents evacuated to Houston. The statistics on their rate of return to the city draw the outline of a phenomenon that can be only partially explained through demographic or survey response data. Empirical techniques may very successfully identify shared characteristics of those who chose to return and those who stayed in Texas, but interviews with those individuals identify trends in thought process and social perception that more brightly illuminate the complexity of interaction between cultural context and human behavior (Chamlee-Wright 2010: 167-9).

Chamlee-Wright presents qualitative methods as superior to quantitative methods in their ability to unearth local and even tacit knowledge. However, it is important to remember that qualitative and quantitative methods suffer from some of the same problems in this regard. Chamlee-Wright evokes the argument most notably advanced by F.A. Hayek (1945) that "most of the knowledge that is relevant for entrepreneurial discovery and economic coordination never presents itself in a form that can be articulated to a central authority" (Chamlee-Wright 2010: 31). In other words, the difficulty of tacit knowledge is that it may not be understood even by the acting individual, rendering explicit communication impossible. While it is true that tacit knowledge cannot be captured in empirical data, it also cannot necessarily be communicated through interviews. Chamlee-Wright correctly points out that "econometric analysis (though useful) is better equipped to tell us what happened rather than why or how" (Chamlee-Wright 2010:35). However, given the dual difficulty of capturing a complex social process in black and white and moving knowledge from the tacit to the explicit realm, this criticism may apply equally well to all data-based analytical ventures, including qualitative methods. 
As further illustration of the overlap of the difficulties across qualitative and quantitative methods, consider that Chamlee-Wright's research team coded the results of their interviews. Ultimately this requires the creation of distinct categories such as might be found in survey or demographic data. For example, if an interviewee brings up their family the response is labeled 'family,' and then further categorized by whether or not the individual is discussing their family's evacuation, exile, or recovery. Once information is coded in this way, it can be used for quantitative analysis along with other data sets. The simplicity with which qualitative data is transformed into quantitative suggests there is not as much of a difference between the two forms as may be thought. Qualitative and quantitative data are not only similar in their limited ability to capture local and tacit knowledge, but can also be used with the same sets of analytical tools.

This observation is not intended to detract from Chamlee-Wright's analysis, but rather to highlight that the line between quantitative and qualitative data is often blurry. The important methodological choice is not between qualitative or quantitative data, but which from a diverse set of data gathering techniques will be the best way to unearth the relevant knowledge. Given the inability to ever perfectly capture people's tacit knowledge of time and place, the researcher must either ignore the important question or select the best, albeit imperfect, method for illuminating individual's personal experiences. The entire book is a testimony to the latter.

For example, it is through interviews that the role of the leadership of the Mary Queen of Vietnam Church comes to light. The rapid response and therefore recovery of the Mary Queen of Vietnam community illustrates one of Chamlee-Wright's key insights, that social capital can overcome the collective action problem of no one person wanting to be the only one in their neighborhood to return. This insight only became evident through interviewees within the community repeatedly bringing up the church in their conversations with interviewers (Chamlee- 
Wright 2010: 25, 57-79). Any other method of gathering data would likely have never unearthed this important discovery.

\section{Monocentrism, Polycentrism, and Disaster Relief}

A central theme of The Cultural and Political Economy of Recovery is the important role played by private and local individuals and communities in recovery. The effectiveness of these private actors stands in contrast to the government provision of disaster relief. Given this, ChamleeWright's research raises important issues regarding the nature and provision of disaster relief and recovery. The standard assumption is that national governments have a key role to play in disaster relief. But Chamlee-Wright's analysis calls this assumption into question by demonstrating that local communities can be more effective at providing relief in the wake of disaster.

The most common argument in support of state provision of disaster relief is its categorization as a public good. ${ }^{2}$ However, to the extent that this is true, this categorization still leaves open the question of the level at which disaster relief is public. Disaster relief may be public at the city or state level, but not the national or global level (see Ostrom 1972, 1983; Aligica and Boettke 2009). Further, if we treat disaster relief as a heterogenous good, which is a realistic assumption given that different communities are affected differently by the same natural disaster, then the public characteristics of disaster relief are likely to be local in nature.

\footnotetext{
${ }^{2}$ Shughart (forthcoming) calls this assumption into question.
} 
Ostrom, Tiebout, and Warren (1961) make the important distinction between "monocentric" and "polycentric" orders. If the provision of a good or service is left in its entirety to a single entity, coercive decision making power is centralized resulting in a monocentric system of governance. In contrast, if the good or service is instead provided by multiple autonomous centers, such as local governments, the system of governance is polycentric. These two types of systems are not geographically exclusive. The city of New Orleans for example falls under the jurisdiction of multiple overlapping layers of governance, with the U.S. government being the most monocentric of these layers and the individual parishes and community organizations being the most polycentric. Similar to the distinction between public and private goods, the difference between mono- and poly-centrism is considered best on a continuum rather than as a binary classification.

There are three essential advantages of monocentric organization (Boettke, Coyne, and Leeson 2011). The first of these stems from the ability of monocentric organizations to generate economies of scale in provision. Positive returns to scale in the production of disaster relief and prevention would then be an argument in favor of housing the right to produce in a more monocentric form of government. Second, monocentric organizations are able to use their monopoly power within their jurisdiction to require compliance, thus minimizing free riding and internalizing externalities to the greatest extent possible. Consider the construction of a levee. Whereas a parish could require only the individuals living within that particular district to contribute to construction, the city of New Orleans could mandate that all residents must contribute tax dollars to the levee project. The third advantage is that if there is only one decision maker, the transaction costs associated with coming to agreement over the best course of action will be non-existent, or at least minimized. However, the strength of this advantage is 
muted to the extent that different layers of governance are required to cooperate with each other. If the federal government is required to have the state government's permission before sending in the National Guard, the cost of decision making is raised and this particular advantage is weakened.

So what are the advantages of polycentric provision of a good that has public characteristics? If the prerequisites specific to each of the aforementioned benefits of monocentrism do not hold, polycentrism becomes more desirable (or at least no worse) as an organizational structure. If returns to scale are negative at the relevant margin, maximizing firm size by imposing a monopolistic structure will increase rather than minimize costs. Similarly, if the nature of the particular good is excludable at some level, even if only excludable to other geographic regions rather than to individual people, the need to coercively protect against free riding becomes insignificant. And, as mentioned above, law or tradition can require even the strongest monopoly to procure consent from other organizations, eliminating the advantage of relatively few agents being involved in the decision making process.

In addition to these limitations on the virtue of monocentrism, polycentric orders have some distinct advantages. First and foremost is the ability of a decentralized organizational structure to capture the specific knowledge of local actors, a theme that emerges repeatedly in Chamlee-Wright (2010). Although public provision of a good at any level of government will be subject to the difficulty of calculating economic value in a non-market environment, the fewer the layers of bureaucracy between consumer and producer, the more grounded the producer's decisions will be in terms of understanding the consumers wants and the value placed on provision of the good (see Mises 1944). 
Perhaps the most compelling case for dispersing decision making power across multiple autonomous groups is the flexibility of polycentrism to adapt to different scales of public goods provision. If the benefits of monocentrism are sufficient to justify a more centralized arrangement of coercive decision making power than currently exists, the smaller polycentric units can contract amongst themselves to build up to a larger scale of provision. So long as contracting is possible, this mechanism allows polycentric orders to capture all the gains of monocentric ordering without sacrificing any of the benefits of the polycentric order, namely access to local knowledge and small scale provision when appropriate. Conversely, monocentric allocations of decision making power crowd out the possibility of smaller scale provision.

Although Chamlee-Wright does not explicitly address the issues of monocentric versus polycentric orders, one of the key benefits of her analysis is that it calls into question the standard view that disaster response and recovery is the exclusive realm of the government. Specifically, her study highlights that the proper scale of disaster relief provision depends not only on the good in question, but also on the unique circumstances facing each relevant locality. Chamlee-Wright shows that the small-scale actions of private actors can be more effective than state-provided activities at understanding these local circumstances and responding appropriately in post-disaster environments. One area of future research is to better understand the different scales and scopes of disaster recovery and reconstruction along the lines discussed above.

\section{Entrepreneurship in Non-Priced Environments}

Economists have a well-developed theory of entrepreneurship in for-profit settings (see Kirzner 1973). At the core of this theory is the central role played by prices and profit and losses play in 
allowing entrepreneurs to engage in 'economic calculation.' Economic calculation refers to the decision-making process of how to best allocate resources among the array of feasible alternatives and involves the determination of the expected value added, or monetary profit, of a potential course of action (Mises 1949: 209-211). The process of economic calculation allows entrepreneurs to weigh the opportunity cost of alternative courses of action and provides continual feedback regarding the allocation of resources.

However, when we move from the realm of for-profit activity to non-profit activity, such as disaster relief, this feedback mechanism is lost (see Boettke and Prychitko 2004; Boettke and Coyne 2008). Disaster relief is non-profit in nature and entrepreneurs in this setting rarely price and sell their output, meaning they are unable to engage in economic calculation. A key theme of The Cultural and Political Economy of Recovery is how entrepreneurs are able to operate in this non-priced environment. Drawing on a variety of concepts form the theory of social networks, Chamlee-Wright explores how social systems can provide feedback in the absence of profits/losses and the pricing of final output. ${ }^{3}$

For example, she highlights how various mechanisms, such as status and reputation within the community, can serve as cognitive tools which allow people to allocate their efforts and resources toward achieving their desired ends. These alternative feedback mechanisms are embedded within the community, providing further support for the value-added of the "cultural toolkit" for economic analysis discussed in Section 2. Chamlee-Wright is careful to point out that these alternative feedback mechanisms are not replacement for the price and profit and loss system in markets. Rather, her point is that in the absence of these first-best feedback

\footnotetext{
${ }^{3}$ See also Chamlee-Wright and Myers (2008) for a discussion of discovery and social learning in non-priced environments.
} 
mechanisms, there are other means available at the local level for people to coordinate their actions to achieve mutually beneficial outcomes.

A related point which emerges from Chamlee-Wright's analysis is the role of "signal noise" and "regime uncertainty" which result from government interventions in the local recovery process. Returning to our discussion of monocentricism in the previous section, the involvement of federal government officials in the recovery process often caused more harm than good. For example, one interviewee noted that FEMA representatives constantly changed and that they "never had the same answer to the same question" while others indicated that instead of providing assistance, FEMA was viewed as yet another bureaucratic roadblock to work around (Chamlee-Wright 2010: 94-6). Other interviewees noted how regulatory rigidities stifled flexibility and fast action in the recovery process (Chamlee-Wright 2010: 142-146).

These examples illustrate two major negative costs of monocentric provision of disaster relief in the context of local non-priced entrepreneurship. First, in relying on large-scale bureaucracies the monocentric provision of disaster relief is clumsy and inefficient. As the previous quote indicates, when different people from the same government agency provide different answers to the same question it creates an environment of uncertainty for local entrepreneurs looking to foster recovery. Second, monocentric orders impose rules and regulations on local communities which necessarily are designed by abstracting from local conditions. As such, these regulations neglect, and oftentimes distort or even destroy, the aforementioned community-level feedback mechanisms—e.g., reputation, status, etc.-which are crucial to facilitating effective cooperation in the recovery process. 
Sociologists have long recognized the important role played by networks as illustrated by Granovetter (2005), who has made clear the value-added of social network analysis for economists. Chamlee-Wright builds on Granovetter's connection by exploring how social network analysis can be applied to disaster recovery. Among other topics, future research should focus on understanding the 'outer limits' of the alternative feedback mechanisms indentified by Chamlee-Wright. For instance, how far can reputation and status extend before they begin to break down? Developing this line of research has important implications beyond disaster recovery and can be applied to a wide array of activities that fall under the categories of 'civil society' and 'social entrepreneurship.'

\section{Concluding Remarks}

The Cultural and Political Economy of Recovery provides a unique and illuminating view of disaster recovery. In doing so it serves as an example of the benefits from exchange across the social sciences, both in the questions it asks and in the methods employed. In addition to the contributions discussed in the above sections, the value of Chamlee-Wright's process-oriented conception of culture can be seen in the diversity of the further avenues for research that it suggests.

First, there is much to develop regarding the process through which culture emerges and evolves. Of particular interest is the feedback loop between culture and economic outcomes. Second, qualitative methods can serve as a complement to standard quantitative techniques which are common in economic analysis. Third, we need to reconsider the costs and benefits of monocentricism versus polycentricism in the provision of disaster relief. Disaster relief is often 
viewed as the realm of government, but one of the main contributions of The Cultural and Political Economy of Recovery is to demonstrate that private individuals at the local level are often better able to engage in effective disaster recovery. Finally, there is work to be done on the array of feedback mechanisms at work in non-priced environments. Social network theory provides a readily available foundation to pursue this line of inquiry which has implications not only for disaster recovery, but for entrepreneurship in all non-priced environments. 


\section{References}

Aligica, P.D. and P.J. Boettke (2009). Challenging Institutional Analysis and Development. New York: Routledge.

Boettke, P.J. (1989). "Evolution and Economics: Austrians as Institutionalists." Research in the History of Economic Thought and Methodology 6: 73-89.

Boettke, P.J. and C.J. Coyne. (2008). "The Political Economy of the Philanthropic Enterprise." In Non-Market Entrepreneurship, eds. G.E. Shockley, P.M. Frank and R.R. Stough, pp. 71-88. Cheltenham, UK: Edward Elgar.

Boettke, P.J., C.J. Coyne, and P.T. Leeson. (2011). "Polycentrism and Gargantua.” Mimeo.

Boettke, P.J. and D.L. Prychitko. (2004). "Is an Independent Nonprofit Sector Prone to Failure?" The Philanthropic Enterprise 1(1): 1-63.

Boettke, P.J., E. Chamlee-Wright, P. Gordon, S. Ikeda, P.T. Leeson, and R. Sobel. (2007). "The Political, Economic, and Social Aspects of Katrina." Southern Economics Journal 74(2): 363-376.

Chamlee-Wright, E. and J.A. Myers. (2008). "Discovery and social learning in non-priced environments: An Austrian view of social network theory." Review of Austrian Economics 21 (2/3): 151-166.

Chamlee-Wright, E. (2010). The Cultural and Political Economy of Recovery: Social learning in a post-disaster environment. New York: Routledge.

Di Tella, R., S. Galiana, and E. Schargrodsky. (2007). "The Formation of Beliefs: Evidence from the Allocation of Land Titles to Squatters." Quarterly Journal of Economics 122(1): 209241.

Granovetter, M. (2005). "The Impact of Social Structure on Economic Outcomes." Journal of Economic Perspectives 19(1): 33-50.

Hayek, F.A. (1945). "The Use of Knowledge in Society." American Economic Review 35 (4): 519-530.

Kirzner, I. (1973). Competition and Entrepreneurship. Chicago: University of Chicago Press.

Mises, L. von. (1944). Bureaucracy. New Haven, CT: Yale University Press. (1949). Human Action. Indianapolis, IN: Liberty Fund, Inc. 
Ostrom, E. 1972 [1999]. "Metropolitan Reforms: Propositions Derived from Two Traditions.” In Polycentricity and Local Public Economies, ed. M.D. McGinnis, pp. 139-160. University of Michigan Press: Ann Arbor, MI.

- (1983). "A Public Choice Approach to Metropolitan Institutions: Structures, Incentives and Performance.” Social Science Journal 20(3): 79-96.

. (1986). “An Agenda for the Study of Institutions.” Public Choice 48(1): 3-25.

Ostrom, V., C.M. Tiebout and R. Warren. (1961). “The Organization of Government in Metropolitan Areas: A Theoretical Inquiry." American Political Science Review 55: 831842.

Shughart, W.F. (Forthcoming). "Disaster Relief as a Bad Public Good." The Independent Review. 